\title{
Nonequilibrium and Nonperturbative Dynamics of Ultrastrong Coupling in Open Lines
}

\author{
B. Peropadre, ${ }^{1}$ D. Zueco, ${ }^{2,3}$ D. Porras, ${ }^{4,5}$ and J. J. García-Ripoll ${ }^{1, *}$ \\ ${ }^{1}$ Instituto de Física Fundamental IFF-CSIC, Calle Serrano 113b, Madrid E-28006, Spain \\ ${ }^{2}$ Instituto de Ciencia de Materiales de Aragón y Departamento de Física de la Materia Condensada, \\ CSIC-Universidad de Zaragoza, Zaragoza E-50009, Spain \\ ${ }^{3}$ Fundación ARAID, Paseo María Agustín 36, Zaragoza E-50004, Spain \\ ${ }^{4}$ Facultad de CC. Físicas, Universidad Complutense de Madrid, Madrid E-28040, Spain \\ ${ }^{5}$ Department of Physics and Astronomy, University of Sussex, Brighton BN1 9QH, United Kingdom
}

(Received 18 July 2013; published 9 December 2013)

\begin{abstract}
The time and space resolved dynamics of a qubit with an Ohmic coupling to propagating 1D photons is studied, from weak coupling to the ultrastrong coupling regime. A nonperturbative study based on matrix product states shows the following results, (i) The ground state of the combined systems contains excitations of both the qubit and the surrounding bosonic field. (ii) An initially excited qubit equilibrates through spontaneous emission to a state, which under certain conditions is locally close to that ground state, both in the qubit and the field. (iii) The resonances of the combined qubit-photon system match those of the spontaneous emission process and also the predictions of the adiabatic renormalization [A. J. Leggett et al., Rev. Mod. Phys. 59, 1 (1987)]. Finally, nonperturbative ab initio calculations show that this physics can be studied using a flux qubit galvanically coupled to a superconducting transmission line.
\end{abstract}

DOI: 10.1103/PhysRevLett.111.243602

PACS numbers: 42.50.Ct, 03.67.Ac, 85.25.Cp

Recently achieved in experiments with superconducting circuits [1,2], polaritons [3,4], and two-dimensional electron gases [5], ultrastrong coupling (USC) is usually linked to the study of discrete systems interacting with cavities, where it is defined as the coupling strength at which counterrotating terms become relevant, the number of excitations (photons) is not conserved, and the rotating wave approximation (RWA) breaks down.

We extend the notion of USC to free space, describing superconducting qubits in open transmission lines. For that we model the atom-light interaction with the spin-boson (SB) Hamiltonian [6,7]

$H=\sum_{k} \omega_{k} a_{k}^{\dagger} a_{k}+\frac{\omega_{\mathrm{at}}}{2} \sigma^{z}+g \sigma^{x} \sum_{k}\left(u_{k}^{*} a_{k}+u_{k} a_{k}^{\dagger}\right)$.

This contains a quasicontinuum of frequencies for the propagating photons, $\omega_{k}$, a two-level system for the superconducting qubit, $\omega_{\text {at }}$, and a realistic set of coupling strengths $u_{k}$ for the specific qubit type. As in the interrupted transmission line [8], the qubit-line system belongs to the Ohmic regime, with a linear spectral function

$$
J(\omega)=\pi \sum_{k} 2 g^{2}\left|u_{k}\right|^{2} \delta\left(\omega-\omega_{k}\right) \sim 2 \pi \alpha \omega^{1} .
$$

The parameter $\alpha=2\left(g_{\text {eff }} / \omega_{\text {at }}\right)^{2}$, quantifying the strength of the SB coupling, is related to the coupling $g_{\text {eff }}$ between the qubit and a resonant cavity made from the same transmission line. While USC effects will be shown in this work for $\alpha \gtrsim 0.1$ or $g_{\text {eff }} / \omega_{\text {at }} \gtrsim 25 \%$, a drastic change in the dynamics is observed at the point $\alpha=1 / 2\left(g_{\text {eff }} / \omega_{\text {at }}=50 \%\right)$ at which both non-Markovian and non-rotating wave approximation effects become relevant in free space.
Our goal is precisely to develop theoretical tools for studying the relaxation and scattering dynamics of a superconducting qubit in an open line, in all coupling regimesweak, USC, and beyond. A proper description of such ongoing and future experiments [9-12] demands theoretical tools that study simultaneously the dynamics of the qubits and the bosons, both in time and space, accurately and without tracing out the line or applying ad hoc decoupling schemes. This goal is achieved using customized matrix product state (MPS) numerical methods that merge ideas from the quantum impurity ansatz [13], matrix product operators [14], and mixed time evolution methods [15]. Our methods rely on the coupled resonator model for the 1D transmission line. Unlike logarithmic discretizations in energy space from numerical renormalization group [16-18] or polynomial discretization MPS [19], the focus lies on precise representations of real space observables, such as the distribution of photons, propagating wave packets or correlations.

In this Letter, apart from confirming this distortion of the electromagnetic field, we compute the spatial arrangement of this "photon dressing" in the transmission line. We then study the dynamics of a qubit excited along the $\sigma_{z}$ direction, proving that the system relaxes locally to a joint qubit-line ground state through the emission of one photon whose frequency agrees with adiabatic renormalization theory [6]. The spatial resolution allows us to determine the mechanism for this relaxation, whereby the qubit-line system, though a closed system [20], provides a bath for its own equilibration by pushing the photon to the far ends of the line, creating around the qubit a region of enlarging quasistationary state. The spatially resolved MPS method 
also allows us to locally engineer the states of the bosonic bath and thus study the scattering of photons by the qubit. Simulations show that the scattering resonances happen precisely at the renormalized frequency of the spontaneously emitted photon, mimicking the physics that has been observed in the weak and strong coupling regimes [9-12]. Finally, an ab initio theoretical model is developed for the coupling strength between a flux qubit interacting and an open transmission line. While so far the qubit-line coupling has been derived perturbatively [21], we develop a theory for arbitrary current strengths. Using parameters from ordinary transmission lines and three-junction qubits, we show it is possible to achieve all coupling regimes of the SB model, making this a suitable platform to test our predictions.

Let us write the interaction between a two-level system and a one-dimensional waveguide of photons,

$H=\sum_{i} \frac{\omega_{0}}{2}\left[\left(x_{i+1}-x_{i}\right)^{2}+p_{i}^{2}\right]+\frac{\omega_{\mathrm{at}}}{2} \sigma^{z}+g \sigma^{x} O_{p, x}$.

This lattice of coupled oscillators is the equivalent circuit for a superconducting transmission line [22,23]; $x_{i}$ and $p_{i}$ are the flux and charge variables and the coupling is $O_{p}=p_{0}$ or $O_{x}=x_{1}-x_{0}$, for charge and flux qubits, respectively, [24]. The ground state squeezing in (3) prevents an efficient MPS description in real space [25]. We thus work in frequency space (1) using the modes of an open chain with $L$ sites or oscillators, with quasimomentum $k=(\pi /(L+1)) \times\{1 \ldots L\}$ and spectrum $\omega_{k}=$ $\omega_{0} \sqrt{2-2 \cos (k)}$. Expectation values of $x_{i}$ and $p_{i}$ are recovered from the Fock operators, $a_{k}$, through an orthogonal transformation. Our model is characterized by the ultraviolet cutoff $\omega_{c}=\sqrt{2} \omega_{0}$ and either the microscopic coupling $g$ or the parameter $\alpha$ in Eq. (2). Thus, all figures show joint scales for $g$ and $\alpha$, the latter inferred from a linear fit to $\omega_{k}$.

Any spin-boson state in the MPS form reads $|\psi\rangle=$ $\operatorname{tr}\left(A_{0}^{s} A_{1}^{n_{1}} \ldots A_{L}^{n_{1}}\right)\left|s, n_{1}, \ldots, n_{L}\right\rangle$, with qubit state $s=0,1$ and photon numbers $n_{i} \in 0,1, \ldots, n_{\max }$ in frequency space. The $A^{s, n}$ are matrices of $\chi^{2}$ complex numbers acting as variational parameters. Typically we found a small "bond" dimension, $\chi=\max _{s, i} \operatorname{dim} A_{i}^{s} \leq 40$, enough. Splitting the Hamiltonian, $H=H_{0}+g H_{I}$, time evolution is simulated using a Trotter formula

$$
|\psi(t)\rangle=\left(e^{-i H_{0} t / 2 N} e^{-i g H_{1} t / N} e^{-i g H_{0} t / 2 N}\right)^{N}|\psi(0)\rangle,
$$

with small time steps $t / N$. Equation (4) describes an algorithm where evolution with $H_{0}$ is exact and evolution with $H_{I} \propto \sigma^{x} O_{p, x}$ relies on a method [7,15] that optimally updates the matrices $A$ for fixed dimension $\chi$.

The ground state is computed through imaginary time evolution as $\lim _{\tau \rightarrow \infty}|\psi(-i \tau)\rangle$. For any coupling $g$ the squeezed vacuum polarizes with a nonzero number of photons in each bosonic lattice site (Fig. 1)and the qubit

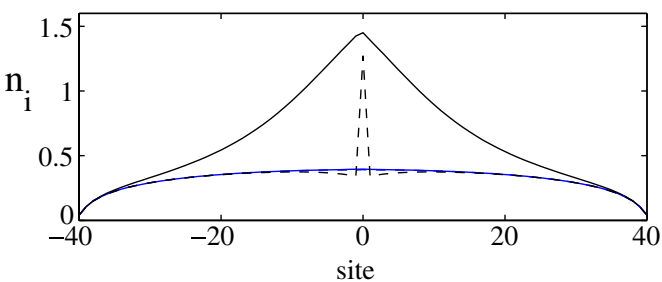

FIG. 1 (color online). Ground state number of photons in each site of the bosonic lattice (3) without a qubit ( $\alpha=0$, blue line below), with a flux qubit ( $\alpha=1$, solid peaked line), and charge qubit ( $\alpha=1$, dashed line). The qubit is located in the middle of the lattice. Note how the perturbation of line due to the flux qubit extends over the whole system.

has some excitation probability $P_{z}=\frac{1}{2}\left(\left\langle\sigma_{z}\right\rangle+1\right)$ [Fig. 2(b)]. This polarization is evident at $\omega_{\text {at }}=0$, where the ground state becomes a Schrödinger cat with two qubit states in the $\sigma^{x}$ basis, and a product of displaced coherent states

$$
\left|\psi_{\omega_{\mathrm{at}} \approx 0}\right\rangle \sim \frac{1}{\sqrt{2}} \sum_{s_{x}=0,1}\left|s_{x}\right\rangle \bigotimes_{k}\left|(-1)^{s_{x}} g u_{k} / \omega_{k}\right\rangle,
$$

that cause a nonzero population of the bosonic modes $\left\langle n_{k}\right\rangle=g^{2}\left|u_{k}\right|^{2} / \omega_{k}^{2}$. Using this ansatz in position space, the distortion is most nonlocal for the $O_{x}$ coupling. In this case the number of photons per local oscillator departs from the vacuum fluctuations all along the line (Fig. 1) and the line develops a small current, $I \propto x_{i+1}-x_{i}$, compensated by a singularity around the qubit.

We have studied the spontaneous emission from an excited qubit which is suddenly coupled to the line $|\psi(0)\rangle=\left|s_{z}=1\right\rangle \otimes_{k}\left|n_{k}=0\right\rangle$. We have found that after a sufficiently long time the state of the system consists of a travelling photon far at the edges of the line, plus a region of the line whose local observables equilibrated together with the qubit. This is first seen for the qubit, whose excitation probability $P_{z}$, relaxes to that of the combined qubit-line ground state, as shown in Fig. 2(b). In the weak and strong coupling limits, $\alpha<0.1$, the relaxation rate is obtained from a master equation, $\gamma=J\left(\omega_{\text {at }}\right) / 2 \propto g^{2}$. For $g \rightarrow 0$, the rate slows down and produces the Gaussian in Fig. 2(b). For $\alpha>0.1$, radiative decay is corrected with an asymptotic excitation probability. Finally, for $\alpha>1 / 2$ the excited state population relaxes even faster to the ground state value, within a time scale $\sim 1 / \omega_{\text {at }}$, deviating from the Markovian law.

At the same time that the qubit equilibrates, so do the photons. Figure 3(a) shows the distribution of photons in frequency space, $n_{k}=\left\langle a_{k}^{\dagger} a_{k}\right\rangle$, a long time after the photons are emitted. The distribution basically consists on one (or less) extra photons imprinted on top of the state of the line in the presence of a qubit. For weak coupling, $\alpha<0.5$, the ground state contains almost no photons and the emitted radiation peaks around

$$
\omega_{\mathrm{eff}}=\omega_{\mathrm{at}}\left(p \omega_{\mathrm{at}} / \omega_{c}\right)^{\alpha /(1-\alpha)},
$$




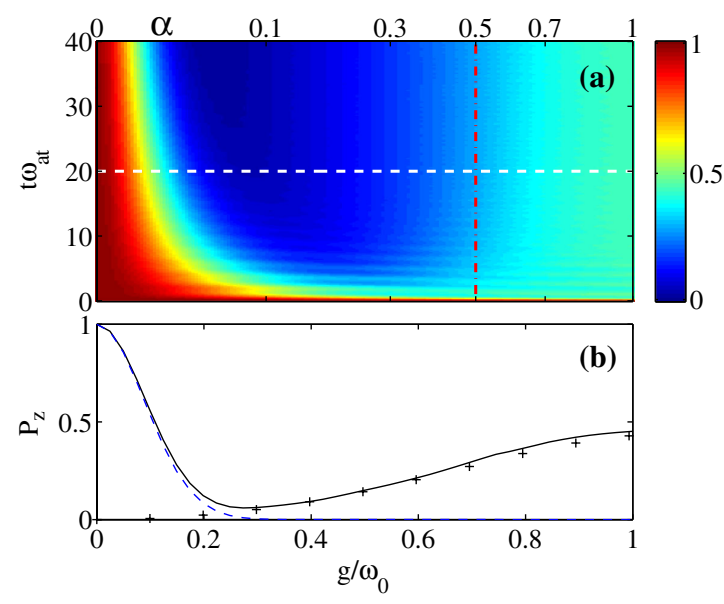

FIG. 2 (color online). Excitation probability, $P_{z}=\frac{1}{2}\left(\left\langle\sigma_{z}\right\rangle+1\right)$, of an initially excited flux qubit in the open transmission line. (a) Full dynamics and (b) final average excitation at the white line $t \omega_{\text {at }}=20$ (black solid line), with the master equation prediction (blue dashed) and the ground state value (crosses). Horizontal axes show both the value of the microscopic coupling $g$ and the parameter $\alpha$ for $L=121$ and $\omega_{\text {at }} / \omega_{0}=1 / 3$. The qubit relaxes to the ground state Bloch vector, but for small $g$ and $\alpha$, the relaxation rate decreases as $g^{2}$ and $P_{z}$ is Gaussian. The vertical line marks the value $\alpha=1 / 2$.

the resonance estimated in Ref. [6], with fit parameter $p=0.5$. For stronger interactions the emitted photon is completely spread in frequency space and $n_{k}$ is close to the result from Eq. (5).

A similar analysis can be done in position space, now studying $\left\langle\psi(t)\left|n_{i}\right| \psi(t)\right\rangle-\left\langle\psi(0)\left|n_{i}\right| \psi(0)\right\rangle$, the difference between the number of photons per oscillator at times $t$ and zero. This is shown in Fig. 4(a) for $O_{p}$ coupling.

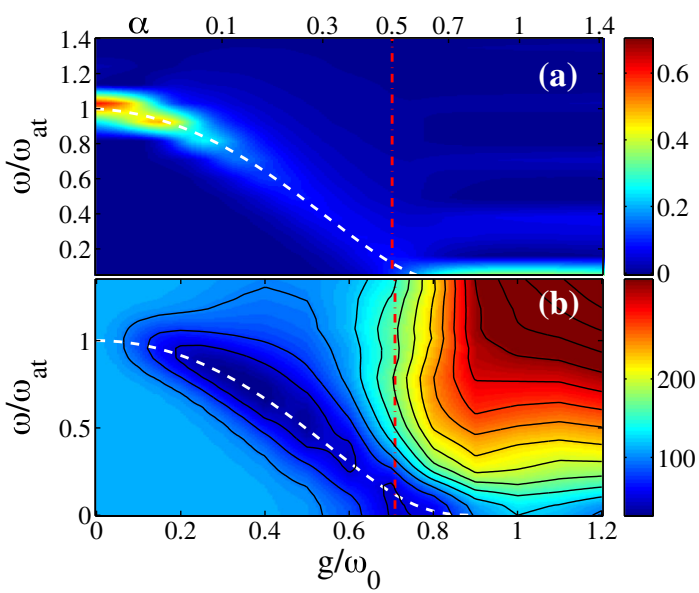

FIG. 3 (color online). (a) Normalized distribution of photons in frequency space vs coupling $g / \omega_{0}$ or $\alpha$, at $t=45 / \omega_{\text {at }}$ after spontaneous emission; RG prediction (6) in dashed line and $\alpha=1 / 2$ line in dash-dotted line. (b) Transmitted photons for a coherent wave packet with average number of photons 1 , as a function of the photon frequency, $\omega$, and the coupling strength $g / \omega_{0}$ or $\alpha$
Note how the travelling photon departs from the qubit at $x=0$, leaving the two-level system and its environment in a local state that is close to the ground state. The qubit-line system, though a closed system, seems to provide a bath for its own equilibration, where the bath are the far away extremes of the line. Naturally, this equilibration is incomplete, as finite size effects give rise to revivals due to the photon reflection at the borders, but it is far from obvious that this finite time equilibration works beyond the weak system-bath coupling regime.

Remarkably, for all values of the coupling, the spontaneous emission properties still dictate the efficiency of a photon absorption process. In other words, the effective frequency $\omega_{\text {eff }}$ also corresponds to the resonances of the qubit-line system when driven by external photons, as shown in Fig. 3(b). This has been verified by studying the interaction of the qubit with a single incident photon. As shown in Fig. 4(b), a photon of frequency $\omega$ is absorbed and reflected by the qubit after a finite interaction time, a process whose efficiency peaks around $\omega \sim \omega_{\text {eff }}$ [Fig. 3(a)]. Above $\alpha=0.5$, the collision is too broad in time and space, preventing the study of scattering coefficients.

Adding a symmetry breaking perturbation, $\varepsilon \sigma^{x} / 2$, to (3) allows us to distinguish different thermalization regimes. The latter can be characterized by the susceptibility in the stationary state, $\chi_{x}=\partial P_{x}(t \rightarrow \infty) / \partial \varepsilon$, with $P_{x}$ the probability to stay in $|1\rangle$ after spontaneous emission. If $\alpha<1 / 2$, the steady-state susceptibility, $\chi_{x}$, matches the groundstate susceptibility of the SB model [8]. This result agrees with the fact that in the perturbative regime a Markovian process cools the qubit to the bath temperature. While the range $0<\alpha<1$ corresponds to the antiferromagnetic Kondo phase [6], above $\alpha>1 / 2$ the Markovian picture breaks down and $\chi_{x}$ departs from the ground-state value. Finally, in the range $\alpha>1$, the qubit-line is in the localized

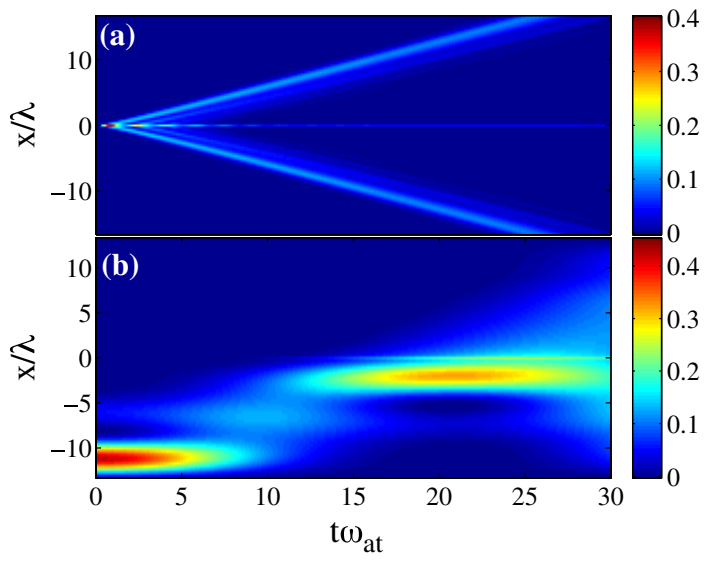

FIG. 4 (color online). Real space distribution of photons relative to the squeezed vacuum. (a) Spontaneous emission from a qubit at $x=0$, with $g=0.475 \omega_{0}$. (b) Scattering of an incoming photon by a qubit at $x=0, g=0.7 \omega_{0}, \omega=0.186 \omega_{\text {at }}$. In both cases $L=121, \omega_{\text {at }}=1 / 3 \omega_{0}$. 


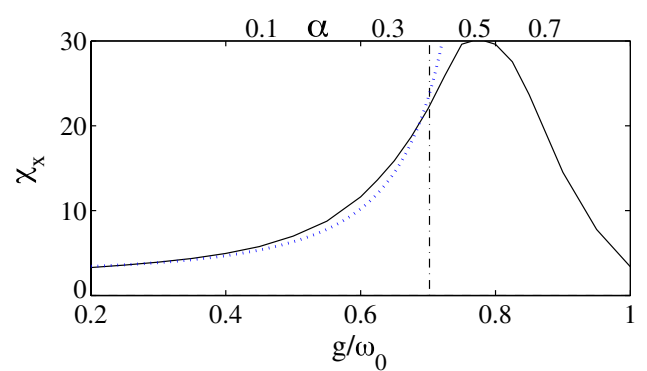

FIG. 5 (color online). Susceptibility of the qubit along the $X$ direction (solid line) when spontaneously emitting a photon while subject to a perturbation $\varepsilon \sigma_{x} / 2$, with a fit to $a / \omega_{\text {eff }}$ (dotted line).

phase, such that the qubit dynamics gets frozen and $\chi_{x}$ vanishes (See Fig. 5).

Let us now discuss how to physically implement the previous model and simulations using a Josephson junction (JJ)-based qubit in an open transmission line. In previous works [21] it was shown that a qubit can be ultrastrongly coupled to a microwave resonator through a derivation that is perturbative in the line-qubit interaction. Our approach regards the qubit as one more element in the discretized transmission line, proving that the coupling strength can be obtained nonperturbatively from the transmission line and the qubit separately.

Following Fig. 6(a), the qubit is regarded as a black-box element coupled to an interrupted resonator. The full Lagrangian $\mathcal{L}=\mathcal{L}_{L C}+\mathcal{L}_{\mathrm{int}}+\mathcal{L}_{q b}^{\prime}$ is expressed in the oscillator, $\phi_{ \pm}:=\phi_{R} \pm \phi_{L}$, and qubit variables $\varphi_{ \pm}:=$ $\phi_{b} \pm \phi_{a}$

$\mathcal{L}_{L C}=\frac{C_{0}}{4} \dot{\phi}_{+}^{2}+\frac{C_{0}}{4} \dot{\phi}_{-}^{2}-\frac{1}{4 L_{0}} \phi_{+}^{2}+\frac{1}{4 L_{0}} \phi_{-}^{2}$,

$\mathcal{L}_{q b}^{\prime}=\frac{1}{4 L_{0}}\left(\varphi_{+}^{2}+\varphi_{-}^{2}\right)+\mathcal{L}_{q b}$,

$\mathcal{L}_{\mathrm{int}}=\frac{1}{2 L_{0}}\left(\varphi_{-} \phi_{-}+\varphi_{+} \phi_{+}\right)$

Note the qubit renormalization and the simple form of the interaction. In practical examples $\varphi_{+}$will not form part of the qubit, but will lock to the oscillator degrees of freedom. This leaves a single operator $\varphi_{-}$that couples only to the antisymmetric mode as in Ref. [21]. Estimating the coupling amounts to computing the matrix elements of $\left\{\varphi_{-}, \phi_{-}\right\}$in the resonator and qubit basis.

These methods have been used for computing the photon-qubit coupling between a transmission line and different flux qubits, in the configurations from Fig. 6(a). As shown in Fig. 6(b), the flux qubit can reach the USC for a reasonable value of the small qubit size $\alpha=0.7$. These results also show that the coupling strength can be changed using qubits where one junction is replaced by a SQUID that adjusts both the gap and the coupling [26-28]. This would allow moving in and out of the USC regime, with (a)

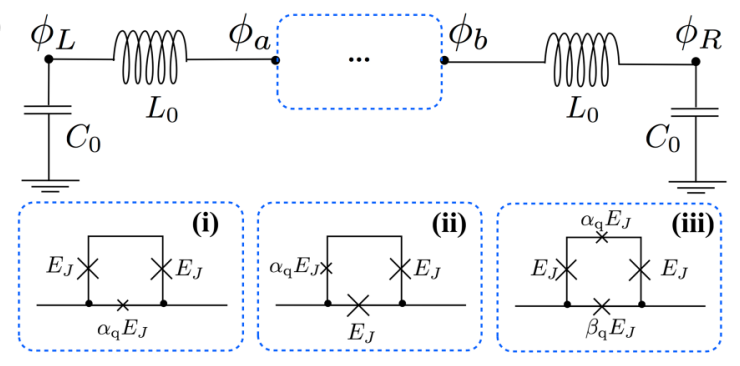

(b)

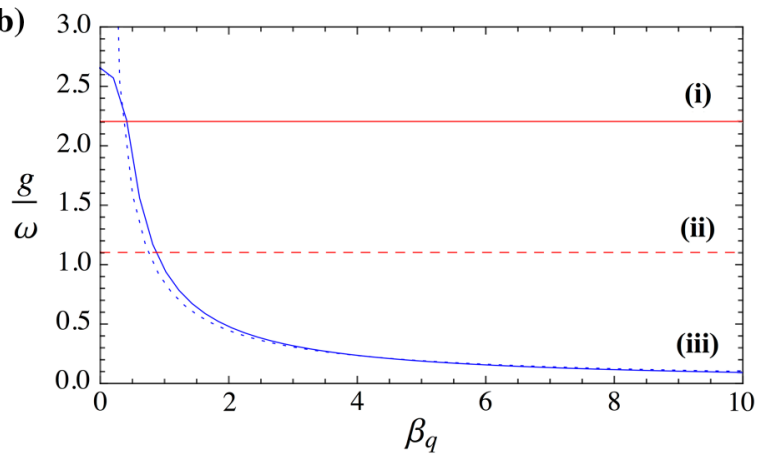

FIG. 6 (color online). (a) Lumped element circuit for a flux qubit ultrastrongly coupled to a microwave resonator by inserting it in the transmission line. (b) Effective coupling strengths for the respective circuits, together with a fit $0.84 / \beta^{0.92}$ (dashed blue line) for the 4-JJ setup, consistent with Ref. [21]. We assume an impedance $Z_{0}=100 \Omega$ and a small junction $\alpha_{q}=0.7$.

the aim of preparing the qubit in excited states and performing the rapid quenches that are needed to do the spontaneous emission experiments. Note also that for a fixed small junction the 4-JJ qubit [21] does not achieve a better coupling than the 3-JJ qubit, and that even an ordinary transmission line can be used to achieved USC (impedance $Z_{0}=30-100 \Omega$ ).

Summing up, this work shows that a flux qubit coupled to an open transmission line implements a quantum simulation of the SB model in the Ohmic regime, with a sufficient range of couplings to cover all regimes: weak, strong, ultrastrong, and localization phase. A numerical method was developed to simulate the physics of such a qubit in the transmission line, including ground state properties, relaxation of the qubit-line system through spontaneous emission, dynamical susceptibility properties, and absorption properties. While the numerical methods provide sufficient quantitative evidence and are consistent with some earlier theoretical predictions, an experiment with superconducting qubits would be the only means to provide a definitive confirmation to some of the predictions shown in this work. The methods put forward in this Letter will allow the study of more complicated systems, such as the correlation functions [12] and nonlinear scattering phases [11] of travelling photons interacting with one or more qubits, or the effective interactions and entanglement dynamics of qubit ensembles in open transmission lines [29]. 
The authors acknowledge support from the European project PROMISCE, Spanish MINECO projects FIS201125167 and FIS2012-33022, CAM research consortium QUITEMAD (S2009-ESP-1594), and Spanish Ramon y Cajal program.

*jj.garcia.ripoll@csic.es

[1] T. Niemczyk, F. Deppe, H. Huebl, E. P. Menzel, F. Hocke, M. J. Schwarz, J. J. Garcia-Ripoll, D. Zueco, T. Hümmer, E. Solano, A. Marx, and R. Gross, Nat. Phys. 6, 772 (2010).

[2] P. Forn-Díaz, J. Lisenfeld, D. Marcos, J. García-Ripoll, E. Solano, C. Harmans, and J. Mooij, Phys. Rev. Lett. 105, 237001 (2010).

[3] G. Günter, A. A. Anappara, J. Hees, A. Sell, G. Biasiol, L. Sorba, S. De Liberato, C. Ciuti, A. Tredicucci, A. Leitenstorfer, and R. Huber, Nature (London) 458, 178 (2009).

[4] A. A. Anappara, S. De Liberato, A. Tredicucci, C. Ciuti, G. Biasiol, L. Sorba, and F. Beltram, Phys. Rev. B 79, 201303 (2009).

[5] M. Geiser, F. Castellano, G. Scalari, M. Beck, L. Nevou, and J. Faist, Phys. Rev. Lett. 108, 106402 (2012).

[6] A. Leggett, S. Chakravarty, A. Dorsey, M. Fisher, A. Garg, and W. Zwerger, Rev. Mod. Phys. 59, 1 (1987).

[7] See Supplemental Material at http://link.aps.org/ supplemental/10.1103/PhysRevLett.111.243602 for more details on the Matrix Product States numerical method and the microscopic model of the physical implementation.

[8] K. Le Hur, Phys. Rev. B 85, 140506 (2012).

[9] O. Astafiev, A. M. Zagoskin, A. A. Abdumalikov, Y. A. Pashkin, T. Yamamoto, K. Inomata, Y. Nakamura, and J. S. Tsai, Science 327, 840 (2010).

[10] A. A. Abdumalikov, O. Astafiev, A. M. Zagoskin, Y. A. Pashkin, Y. Nakamura, and J. S. Tsai, Phys. Rev. Lett. 104, 193601 (2010).

[11] I.-C. Hoi, C. M. Wilson, G. Johansson, T. Palomaki, B. Peropadre, and P. Delsing, Phys. Rev. Lett. 107, 073601 (2011).
[12] I.-C. Hoi, T. Palomaki, J. Lindkvist, G. Johansson, P. Delsing, and C. M. Wilson, Phys. Rev. Lett. 108, 263601 (2012).

[13] A. Weichselbaum, F. Verstraete, U. Schollwöck, J. I. Cirac, and J. von Delft, Phys. Rev. B 80, 165117 (2009).

[14] B. Pirvu, V. Murg, J. I. Cirac, and F. Verstraete, New J. Phys. 12, 025012 (2010).

[15] J. J. García-Ripoll, New J. Phys. 8, 305 (2006).

[16] F. B. Anders and A. Schiller, Phys. Rev. Lett. 95, 196801 (2005).

[17] F. B. Anders and A. Schiller, Phys. Rev. B 74, 245113 (2006).

[18] P. P. Orth, D. Roosen, W. Hofstetter, and K. Le Hur, Phys. Rev. B 82, 144423 (2010).

[19] J. Prior, A. W. Chin, S. F. Huelga, and M. B. Plenio, Phys. Rev. Lett. 105, 050404 (2010).

[20] A. Polkovnikov, K. Sengupta, A. Silva, and M. Vengalattore, Rev. Mod. Phys. 83, 863 (2011).

[21] J. Bourassa, J. M. Gambetta, A. A. Abdumalikov, O. Astafiev, Y. Nakamura, and A. Blais, Phys. Rev. A 80, 032109 (2009).

[22] B. Yurke and J.S. Denker, Phys. Rev. A 29, 1419 (1984).

[23] M. Devoret, in Les Houches, Session LXIII, edited by S. Reynaud, E. Giacobino, and J. Zinn-Justin (Elsevier, New York, 1995), Chap. 10, pp. 351-386.

[24] Simulations have been done using both couplings, without relevant differences except where explicitly noted.

[25] The entanglement generated by the coupling leads to a large number of photons $n_{i}=\frac{1}{2}\left(x_{i}^{2}+p_{i}^{2}-1\right)$ that demand a large cutoff to represent the state faithfully, even without a qubit.

[26] F. G. Paauw, A. Fedorov, C. J. P. M. Harmans, and J. E. Mooij, Phys. Rev. Lett. 102, 090501 (2009).

[27] J. Friedman, V. Patel, W. Chen, S. Tolpygo, and J. Lukens, Nature (London) 406, 43 (2000).

[28] J. E. Mooij, T. P. Orlando, L. Levitov, L. Tian, C. H. van der Wal, and S. Lloyd, Science 285, 1036 (1999).

[29] A. Gonzalez-Tudela, D. Martin-Cano, E. Moreno, L. Martin-Moreno, C. Tejedor, and F. J. Garcia-Vidal, Phys. Rev. Lett. 106, 020501 (2011). 